\title{
Extending Instant Messaging to Provide Pervasive Personal Communication
}

\author{
Wei Li, Fredrik Kilander and Carl Gustaf Jansson \\ Department of Computer and Systems Sciences, \\ Swedish Royal Institute of Technology \\ \{liwei,fk, calle\}@dsv.su.se
}

\begin{abstract}
This paper proposes a personal software architecture to provide a user with a pervasive communication capability. The core of this architecture is a Personal Communication Server (PCS) which bridges between different Instant Messaging networks and extends them with a unified access to the user. A SIP-based implementation of such a communication server is presented, which solves various interoperability problems, and also provides many value-added features, e.g., extended user presence with location information, adaptive communication, better privacy protection, etc. This implementation enables various existing SIPcompatible resources to be re-used and thus simplifies the construction of the proposed software architecture.
\end{abstract}

\section{Introduction}

The Internet has indeed changed the way we communicate. For some time, E-mail has virtually replaced traditional letters for many of us. It provides a far more convenient and economical way for people at different physical locations to exchange information efficiently. More recently, an alternative, Instant Messaging (IM) (as well as its variants of SMS and MMS in cellular networks) has gained in popularity. More advanced than e-mail, IM supports real-time conversation between the communication parties (socalled buddies). In addition, it offers "presence awareness" so that a user can see her buddies' status (e.g., idle, busy, away, etc.), and then determine how to communicate with them. Much of this presence information can also be provided by the IM application itself thus relieving a user from manual specification. Other lately integrated powerful features, such as Smileys and the support for audio and video, have further enhanced the plain text chatting into a more user-friendly activity.
With increasing Internet ubiquity, IM applications (commonly referred to as Instant Messengers) have gained popular acceptance, and are becoming a type of pervasive applications in our everyday lives. People are expecting more integrated functions, e.g., to control their home electronic appliances, or conduct ebusiness anywhere using their messengers on mobile devices. Consequently, IM will probably become a dominant feature in future personal communication. However, today's IM systems are not ready for such an important role due to many existing problems with them.

This paper addresses some critical problems with today's IM systems and applications, and proposes a personal software architecture as their solution. The core of this proposal is a Personal Communication Server (PCS), which bridges between different IM systems, and extends them to present unified access to the user. A SIP-based implementation of such a Personal Communication Server is presented. It translates information between the proprietary IM networks and the standard SIP protocols supported by the personal infrastructure. By solving many interoperability problems regarding SIP in this PCS implementation, we have succeeded to access various existing SIP-compatible resources (as well as the public IM services) with value-added features. This achievement simplifies the construction of a Personal Communication System, enabling us to leverage rich existing third-party resources to provide a user with pervasive personal communication.

The rest of the paper is organized as follows. The following section 2 enumerates a number of related problems with the current IM systems, together with our motivations of providing personal extensions. Then as a proposed solution, we present in section 2 a personal software architecture, wherein the Personal Communication Server plays the core role to solve various interoperability problems. Section 4 gives an introduction of SIP and SIP SIMPLE which we adopted as general communication protocols. Section 5 
elaborates our implementation of a SIP-based Personal Communication Server, and its value-added features. Finally, we give some discussion on related work in section 6, and end with our conclusions and future work in section 7 .

\section{Problems with Existing Systems}

Although various IM applications have gained popular acceptance, there are many problems in the current IM systems, which restrict them from being pervasively useful to the end users. Here we first give an enumeration of the issues we think most critical, and then present our motivation on how to attack them.

\subsection{Interoperability Difficulty}

There are too many IM service providers, each with its own protocols; therefore, they cannot communicate with each other directly. Since ICQ was introduced as the first general instant messenger in 1996, the list of online IM services keeps growing. Today, the most popular IM applications are Microsoft's Windows MSN Messenger (MSN), Yahoo! Messenger (YIM), and AOL Instant Messenger (AIM). With the recent explosion of Voice over IP (VoIP) in IM, other newcomers, such as Skype and very recently Google Talk, are attracting more and more users. Besides them, many local IM providers have also succeeded in satisfying targeted users with more specialized features. An inexhaustive list of the existing IM protocols and their supported clients can be found at [1].

The interoperability problem has led users to run multiple instant messenger applications simultaneously in order to be available on several IM networks. Alternatively, they can use some universal client, like Gaim [2] and Trillian [3], which supports many protocols. However, then some more advanced features, such as audio, video, and file transfer functions are subject to be unavailable, since they are specific to each IM network.

\subsection{Poor Mobility Support}

Not every popular IM service provides a client for mobile devices such as PDAs and mobile phones. Even with the available mobile clients, the user could easily find them less useful as they are often ported to a mobile device as a simple copy of their PC version, which has little consideration for the user's mobile situation.

\subsubsection{Singular Online Instance}

The user needs to use the same account from multiple devices simultaneously, so that she can use any device which is handy to her at the time to handle her communication, e.g., to answer a voice call. Unfortunately, most popular IM services (except for Skype) only support a single online instance per account, which means a Sign-In action will force another client, currently connected with the same account, to sign out automatically.

\subsubsection{No Adaptability Support}

The current IM applications spend lots of energy on providing a user-friendly interface; however, there is no support for advanced adaptations, which however often are critically important for mobile users. For example, one would probably not want to respond to massive amounts of Instant Messages and multimedia calls when using a low bandwidth connection on an expensive network such as GPRS. However, the user may still be willing to give out presence information e.g., to her family members. Instant Messages with the current systems always arrive at the user's device (which already results in a cost) unless the client is signed out of the IM services, but then the presence information is no longer provided.

A more appreciated solution would be for the IM service to store the messages and notify the user to handle them later, e.g., when a Wireless LAN is available. Another adaptation might be that the incoming multimedia call could be rendered by nearby computing facilities with better interaction support (e.g., a big touch screen) than the user's current mobile device. This would also save the battery for the mobile device. Such adaptabilities for making optimal use of available resources would give great enhancement to mobile communication; however, they are simply not possible with the current IM systems.

\subsection{Poor Privacy and Security Support}

Before communicating with their buddies, each user has to sign in to the IM network (some specific server). In most IM systems, every message is sent to this server, and then be delivered to the recipient. While some systems support data encryption, they do not provide end-to-end data protection between the communicating parties. Therefore, the communication server knows every detail of the conversation, from the communication addresses of all parties to the content of every Instant Message. This makes the current public IM systems unsuitable for delivering confidential and sensitive information. 
In addition to the above, other problems have also plagued users, such as the need to keep upgrading the client application when the proprietary communication protocol changes. In particular, people often find themselves unable to sign in to the IM servers, and thus cannot communicate with their buddies even if they are online. This denial of service may be caused by many reasons: network address translation (NAT), the maintenance of the IM servers, or attacks by malicious Internet users (so-called hackers). Naturally, service failures can be very annoying for many users who are used to and rely on IM services to maintain contact with their buddies.

\subsection{Motivations}

We think the main reason for the above problems is the lack of control over the IM services by the end user. The user is confined to the features provided by the client application, which only allows proprietary protocols and excludes others. However, this should not be necessary as an IM service mainly helps a user for user account authentication, address resolution, and message routing among the users' devices. Its essential work is to provide registered users a directory service since capturing and rendering media data are handled by user mobile devices themselves. Given that the user has spent money on their computing devices, and is paying for their Internet communication, the extra requirement of being bounded to the specific IM services seems unreasonable. Therefore, if the main purpose of using IM systems is to stay in contact with family members, friends, and colleagues, rather than to receive other information (e.g., commercials) provided by IM services, then it would be more meaningful to have a controllable, local server (e.g., for personal or group use) rather than general-purpose servers which do not provide information privacy.

As computing resources become cheaper and pervasive, it becomes less difficult to provide an excess resource to run a server for just personal use. For example, some of today's residential wireless routers, like ASUS's WL-500g, can be extended to act as a Call Center by running an Asterisk PBX server from a USB hard drive plugged in [30]. Typically, a home PC could host a communication server, or a small group (e.g., family members) could share a computer to facilitate their communication.

\section{A Personal Software Architecture}

We propose a Personal Communication System ${ }^{1}$, a personal software architecture, to handle communications for each individual user. The core of this architecture is a Personal Communication Server (PCS) which bridges between different Instant Messaging networks and extends them with unified access to the user. Based on the PCS, a number of different communication entities form such a Personal Communication System, providing a user with pervasive communication capability. Similar to a general-purpose public IM server, the PCS takes the responsibility for delivering messages among different communication entities. Beyond this, the PCS can identify various communication entities and take different actions for the user utilizing all available resources.

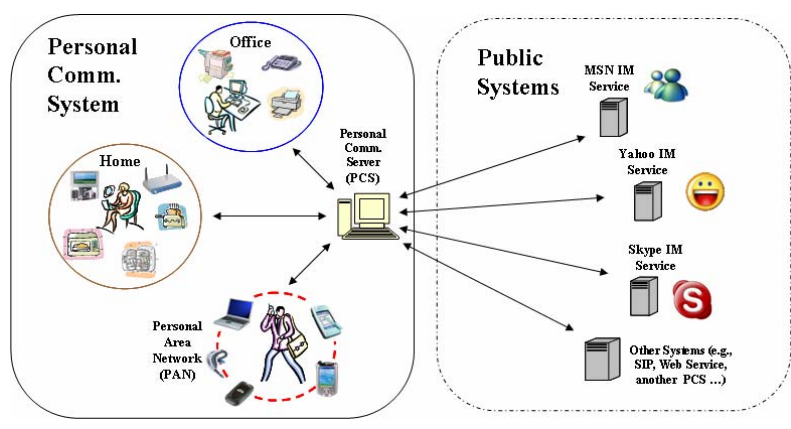

Figure 1. A Personal Communication System

As depicted in Figure 1, different communication entities are divided into two general systems: personal vs. public. The Personal Communication System consists of computing resources controlled by the user and may contain different sub-groups: the user's wearable devices (e.g., in a PAN) provide immediate support for the user's communication, and resources distributed in different physical places (e.g., in user's office and home), which may also contribute to the user's communication in different ways. For example, they could provide storage to record incoming calls and messages when the user is not available. In general, the PCS helps to coordinate different communication entities to provider better communication and interaction support for the user.

\footnotetext{
${ }^{1}$ The Personal Communication System is the proposed personal software architecture and should not be confused with the Personal Communication Server (PCS) which is the core component of the Personal Communication System.
} 
On the other hand, the PCS acts as a single and persistent representative of the user to outside public systems, hiding the internal details of the Personal Communication System from other parties. In the prototype we have implemented, the PCS can connect to most popular IM networks (our implementation currently supports YIM, MSN and Skype but other extensions may also be added if needed). To each of these IM systems, the PCS appears as an ordinary IM client. The PCS can then redirect messages to the user's current devices, and should also utilize available resources to facilitate their communications.

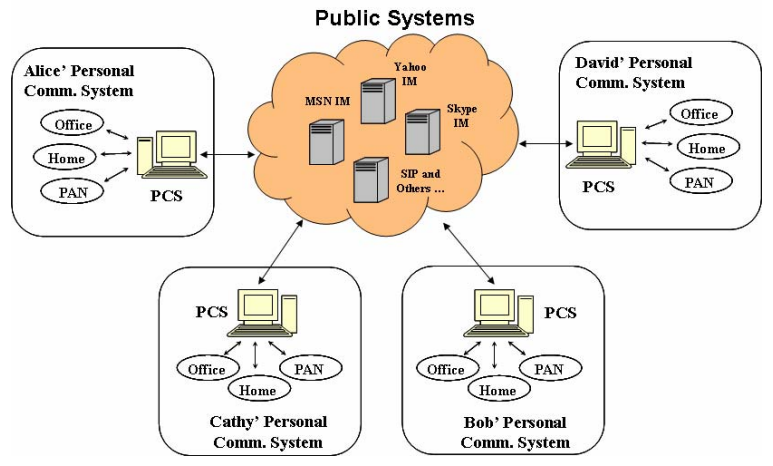

Figure 2. Peer-to-Peer Buddy Network

A number of inter-connected PCSs can form a peerto-peer buddy network, as each extends their individual Personal Communication System. This buddy network can be constructed based on the public IM networks or using direct communication between each PCS. The later solution removes the constraints caused by the use of proprietary protocols, and offers better user privacy protection as information is delivered directly between individual PCSs without any intermediary, such as the third-party IM servers.

To provide an open solution and avoid "reinventing the wheel", the Session Initiation Protocol (SIP) [4] and its extensions have been adopted for direct inter-PCS communication (as well as for internal Personal Communication System). Our decision is based on the fact that SIP has been widely accepted as an open standard for Voice over IP (VoIP) and multimedia conference applications. By using SIP, the user has a wider selection of alternatives and may choose their favorite client applications (with respect to the supported devices and software platforms) from a rich set of existing resources. Thus, the users have greater flexibility on constructing their Personal Communication Systems than being in the confines of a certain commercial product. In the next section, we will give a brief introduction to the communication mechanisms provided by SIP and its extensions.

\section{SIP-based Communication Support}

The Session Initiation Protocol (SIP) is an application layer control (signaling) protocol for creating, modifying, and terminating sessions with multiple parties [4]. A typical session establishment involves a triple-message delivery: A session initiator (SIP User Agent/UA) sends an INVITE request to its governing SIP network. The request is then routed by SIP Proxies (each representing an intermediary SIP domain) until it reaches the receiver's SIP UA. An OK message is sent back to the initiator if the receiver is willing to accept the session in the request. Upon receiving an acknowledgement (ACK) message from the initiator, a session is established, and the media communication begins directly between two endpoints. The media communication parameters are carried as the content of the initial INVITE request (using the Session Description Protocol [5]), but could be modified in the OK response. By sending INVITE request, communication participants with different capabilities could negotiate (or re-negotiate) to determine their mutually preferred communication means.

To add the capability of transferring both real-time Instant Messages and asynchronous information such as presence information. The IETF working group, SIP for Instant Messaging and Presence Leveraging Extensions (SIMPLE) has defined a support of standard Subscribe/Notify/Publish mechanism [6] using three components: A Watcher subscribes to a Presence Agent for its presence information, a Presence User Agent updates presence changes to the Presence Agent, and this information will then be notified to the Watcher as asynchronous updates.

\section{Implementing Personal Communication Server (PCS)}

It is conceptually easy to map the SIP and SIP Presence Framework architecture to our Personal Communication System design. We have implemented the PCS as the persistent representative of a user based on a SIP proxy server with SIP Presence support. Thus, we can provide SIP standard access (multimedia calls, Instant Messages and presence queries) from the buddy network.

We can also deploy various SIP clients on the user's devices to receive and send SIP messages, as well as provide user presence information to the buddy network through the PCS. Therefore, by making use of rich existing SIP client applications, the construction of a Personal Communication System is rather easy. 
However, it turned out we had to solve many problems to enable the existing resources pervasively usable.

\subsection{Solving SIP Communication Problems}

The following subsections present some SIPspecific problems we have discovered during our examination of various existing SIP resources.

\subsubsection{Interoperability Difficulties}

Compared with SIP, SIP Presence Framework is a relatively new standard and has not been widely supported. We have tested two SIP Presence compatible IM clients: Microsoft Windows Messenger (from v4.6 to v5.1) [7] and Microsoft Portrait (PC \& Pocket PC v2.2 and v2.3) [8], both are based on Microsoft's Real-Time Communications (RTC) client API [9]. Two incompatible issues have been discovered:

(1) Some SIP messages (e.g., the Presence Notifications) from these clients do not go through the SIP server (PA) as defined in the Presence Framework. Instead they are sent directly to the requesters (Watchers). This breaks our model that no direct communication with the user's device occurs from external visitors except via PCS mediated multimedia sessions. To overcome this problem, we had to intercept such messages and modify them to point to the PCS (i.e., forging PCS as the message sender or receiver), so that every message will arrive at the PCS before being delivered to other systems.

(2) Different Presence Information Data Formats (PIDF) are supported by different IM clients, e.g., the RTC-based clients support XPIDF [10] which is rather old, it has recently been replaced by PIDF [11]. Thus, the PCS has to convert the information between different clients.

\subsubsection{NAT Traverse}

SIP suffers from communication problem caused by Network Address Translation (NAT). Unfortunately, NATs are commonly used by Local Area Networks (LAN), such as Wireless LANs, as well as wide area networks. Applications behind the NAT use a private IP address which is only valid internally within the same NAT zone. SIP clients will use this local IP address in messages, thus providing false routing paths for replies. Moreover, a reply messages from outside the NAT using such a private address as its destination will not be received by the internal client, because the destination is not routable from outside. Without external help, two internal clients behind different NATs, messages can not deliver directly between them.
To solve this problem, we support symmetric NAT traversal. Here, an external host that receives a message can send message back to the internal host (along the same physical routing path). We assume the PCS has a public IP address, then if the user's SIP client is behind a NAT; it sends a SIP request (e.g., register) first, providing the address of the PCS (to the NAT server), thus will then be able to receive subsequent SIP messages. This solves the problem of SIP NAT traverse for symmetric NATs.

For media data communication using Real-Time Protocol (RTP) [12], Simple Traversal of UDP over NAT (STUN) [13] is proposed as a standard solution. A STUN server helps a SIP client to replace the private IP address and port in its SIP/SDP message with the NAT mapped public IP address and port. This can be done by the SIP client without the involvement of the PCS. However, this solution adds some overhead, e.g., STUN support is required on the client side, and a STUN server needs to be available. Moreover, it will not work for symmetric NATs. In such case, the PCS can establish a tunnel between two SIP clients behind different NAT zones (in the worst case). As the PCS has a public IP address, it can communicate with other internal clients (as long as the first request is initiated from an internal client). The SDP indicates that the media data is to be tunneled via PCS itself or a third public IP host (which will forward the received data to the corresponding receiver(s) respectively).

\subsubsection{Media Format Compatibility}

In addition to the IM function, we also planned to support VoIP since SIP was originally designed for it. Then we discovered the compatibility problem also exists at the media format (CODEC) level, although normally this should be solved by SIP using SDP. The issue we found is that the GSM CODEC in applications using the RTC API does not conform to the most used GSM 06.10 standard [14], it uses an incompatible frame format [15] so should really be identified as Microsoft's GSM format. However, the RTC client does not distinguish this in its SDP message which confuses others.

To avoid this confusion, the PCS can help to identify RTC-based SIP clients, and then correct their announcement of GSM support to be MS GSM in their SDP message to other clients that actually do support the standard GSM format. In addition, our PCS can also transcode the media data, e.g., to use a low bandwidth CODEC to reduce network traffic or to communicate with a client using unsupported CODECs. 


\subsection{Providing Value-added Features}

Based on the solutions presented in section 5.1, we have implemented a prototype PCS. The current prototype is implemented in Java. Its extension parts to the public IM networks are built on our modifications of some open-source Java libraries: JMML [16] for MSN, jYMSG [17] for YIM, and JSkype [18] for Skype. For SIP communication, a JAIN SIP API [19] implementation, NIST-SIP v1.2 [20] is used.

We have then tested various SIP clients on different user devices, specifically, Microsoft's Windows Messenger, Microsoft's Portrait, and Xten's X-Lite [29] on PCs; and Portrait for PocketPC on the HP iPAQs (model H5550). Each of them has succeeded to connect to and communicate through the PCS, and can thus be used to form a Personal Communication System. We can then use any of these clients to send and receive Instant Messages to any other IM network through the PCS. In addition, the PCS can identify the different clients and help to solve many problems, e.g., various compatibility issues; therefore, we can also provide many value-added features which are not possible with the original IM clients.

\subsubsection{Transparent Cross-Network Access}

By extending the access to different IM networks, a PCS allows its user to maintain contact with her buddies from any of these networks. The user is able to see the presence of her buddies and to communicate with them in a transparent way without bothering to know via which specific network(s) they are currently connected.

To simplify the management of the buddylist on the user's terminal device, the PCS provides a one-tomany mapping so that each single buddy presented on the user's terminal device may be associated with multiple physical accounts on specific networks. For example, a buddy named Beichuang may have both YIM and MSN accounts (e.g., beichuang@yahoo.com \& beichuang@msn.com). The person appears as a single buddy to the user; however, an Instant Message to him may be delivered through different IM accounts by the PCS depending on each one' presence status.

\subsubsection{Extended User Presence with Location Information \\ The use of PCS also adds new possibilities on providing user presence information. A user can present her status to different IM networks simultaneously with extra information such as location. As shown in Figure 3, user beichuang's symbolic location "At Home" (together with a timestamp) is seen by his buddy (beichuang2000) through different}

channels (YIM, MSN and Skype accounts). The physical location information can be acquired using different location detection technologies [21]. Here in our example, a GSM Detector application runs on the user's Smart Phone (SonyEricsson P800), constantly monitoring the connected Cell-ID and its radio signal strength, and reports changes to her PCS over a GSM/GPRS connection. The PCS will map the CellID into a symbolic location and longitude/latitude coordinates as well. Then the user's location can be displayed on different maps to authorized buddies (Figure 4). The mapping table can be easily compiled by the user themselves with the Cell Detector application. Additionally, the user is allowed to modify her presence information manually.

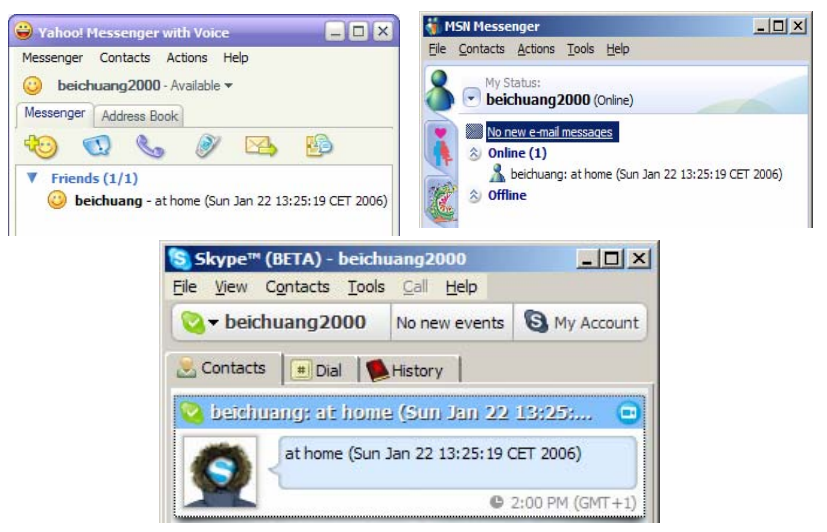

Figure 3. PCS extends ordinary IM presence with symbolic location

The PCS also allows the user to provide different presence towards different IM networks, or even separate ones for each distinct buddy (using SIP) ${ }^{2}$. This feature gives an excellent support for user privacy protection, allowing the user to control who will see what, even in fine-grained details.

Instant Messaging and presence can be handled separately by the PCS, thus, the user can easily achieve the setting of "blocking Instant Messages and multimedia calls while keep presence provisioning", which we mentioned as a useful low-cost setup (in Section 2.2.2.) for mobile users, however has not been supported by any of the current IM systems.

\footnotetext{
2 This feature is only possible towards buddies supporting SIP SIMPLE, and not possible with other buddies using the public IM networks due to the constraints with their IM services.
} 


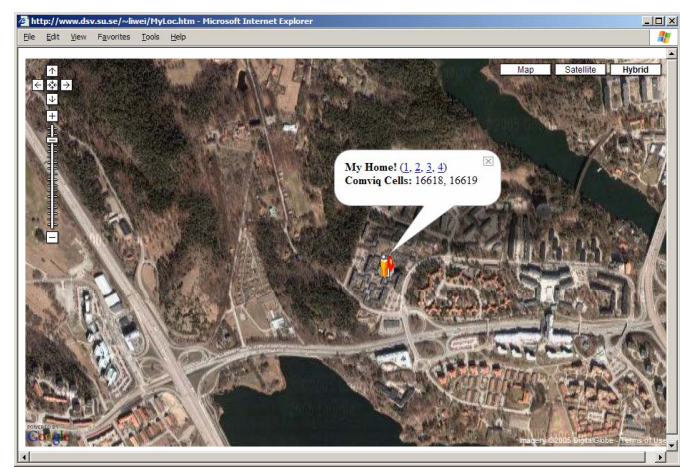

(a) Google Satellite Map

Text: My Home! (extra 4 map links)

Comviq Cells: 16618, 16619

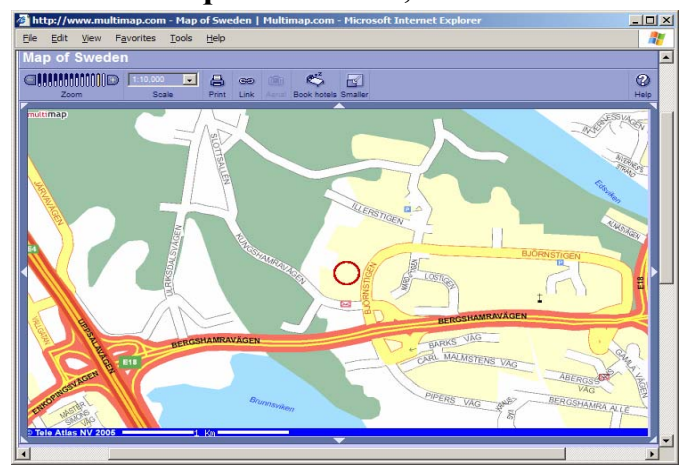

(b) Map with Multimap.com

Figure 4. PCS displays user location with online maps

\subsubsection{Communication Delegation}

The PCS can handle the incoming communication requests for the user when she is not available (e.g., when using a low-bandwidth connection). The PCS will reply with a corresponding busy response (in text or voice) on behalf of the user, and store the missed text and voice messages. When the user returns back to available (e.g., regains a connection with the PCS within a Wi-Fi hotspot area), she will then receive an Instant Message from her PCS, indicating the amount of missed messages grouped with their senders (e.g., "10 missed IMs \& 1 voice message from Smith"). This message also comprises an URL which provides the message contents (voice messages are further presented as URLs). Thus, the user can avoid the traffic load incurred by uninteresting messages.

In our current implementation, a SIP softphonebased Answering Machine is developed using an opensource Java Sound library [22]. It answers the requests redirected from the PCS, and stores the messages into files (each voice call is saved as a separate file while text messages are grouped by their senders). For online storage of the messages, we have built a very simple application (only respond to the HTTP GET requests) just to satisfy our needs with the minimal resources cost. The user's available status is determined so far by only checking her presence from the mobile clients. We are working on auto-adaptation by utilizing the connection status reported from wearable devices.

\subsubsection{Providing Pervasive Contact to Buddies}

There are three alternatives for a buddy to contact a user who has a PCS: using the public IM networks; using inter-PCS SIP communication through the buddy's own PCS; or direct communication (using SIP) with the user's PCS. The last case gives the least constraints to the buddy as he can use any standard SIP softphones to contact the user's PCS, while the user can still enjoy the value-added features. We think this alternative could be very useful for those people who do not have any IM accounts.

\subsubsection{Other Useful Features}

By using the PCS, we have also achieved a number of other useful features:

- Remote Monitoring: We have deployed some SIP softphone clients on some specific places (e.g., the user's home and office), and setup their mode to always accept calls. Then the user can call these different places remotely to see who are there and hear what they are saying. This spy-function is not publicly accessible since all the incoming calls are mediated by the user's PCS which will block unauthorized visitors.

- Combining Different Clients: Sometimes, the user may not find a single suitable client, but instead a number of choices, where each meets some specific requirements. For instance, as we found MS Portrait supports a SIP compatible IM function, but does not offer standard VoIP (only proprietary media formats on $\mathrm{iPAQ}$ ), while other SIP softphone provides VoIP but no IM support. In this case, we can run both of them on the user's device, and the PCS can distinguish them and deliver the corresponding requests to them respectively.

\section{Related Work}

Numerous efforts have been spent on enabling universal IM communication. Multi-protocol IM clients, such as Gaim and Trillian as the most notable, attempts to combine many disparate protocols into a single, unified client. Other more promising work attempts to create a unified standard for instant messaging. In addition to IETF's SIP and SIMPLE, other distinguished standards are open XML-based 
XMPP (Extensible Messaging and Presence Protocol), more commonly known as Jabber [23] and OMA's (Open Mobile Alliance) IMPS (Instant Messaging and Presence Service) [24]. The later was created specifically for mobile devices. However, most of them did not succeed in unifying the existing IM providers; instead, each ends up as a new proprietary protocol.

More specifically in ubiquitous computing, K. Elkhatib et al. [25] has demonstrated using a Personal Agent (also implemented with SIP) to provide better performance and interaction support for a mobile user to handle incoming multimedia calls in a resource-rich environment, also with considerations of the user's preference and available services. Stefan Berger and Henning Schulzrinne et al. elaborated conceptually on how ubiquitous computing systems can be constructed using SIP and other standard protocols [26]. Additionally, Instant Messenger applications have been commonly used as prototype examples, such as the Lemming IM in Confab [27] used to present how to add user privacy control to existing applications. However, the IM in these works only serves as a proof-of-concept.

\section{Conclusions and Future Work}

This paper presents our efforts on extending IM systems to provide a user with pervasive communication. To achieve this goal, we proposed and implemented a Personal Communication Server (PCS) based on open standards (SIP and SIMPLE). With this PCS, various existing software resources can be easily re-used to assemble a Personal Communication System which enables the user a pervasive access. In addition, the user gains more control of their communication, thus offering more personal privacy protection. Many value-added features are also provided to further facilitate the user's communication. Based on these, we can make the existing IM technology more useful to the ordinary end users.

As the future work, we plan to test some emerging SIP IM clients more devotedly compliant with the SIP SIMPLE standards, such as the Ineen's PocketPC client [28] based on Xten's eyebeam SIP API [29]. On the other hand, we have also started building our own mobile clients, e.g., porting SIP softphones to smart phones, more functions will then be integrated, such as, enable capturing and reporting the device status (e.g., connectivity, battery power, memory usage, etc.). With rich client context information, the PCS can provide more advanced adaptations. We will also compare two different strategies on deploying the decision-making logic: on the client side, or the server side, with more complex user scenarios. We suppose a combination of these two strategies could provide a better communication performance.

\section{References}

[1] http://en.wikipedia.org/wiki/Comparison_of_instant_mes sengers

[2] http://gaim.sourceforge.net/

[3] Cerulean Studios, Trillian, http://ceruleanstudios.com/

[4] J. Rosenberg, et al. SIP: session initiation protocol. RFC 3261, IETF, June 2002.

[5] M. Handley, et al. SDP: session description protocol. RFC 2327, IETF, April 1998.

[6] J. Rosenberg. A presence event package for the session initiation protocol (SIP), RFC 3856, Aug. 2004.

[7] Microsoft, http://www.microsoft.com/windowsxp/using/ windowsmessenger/default.mspx

[8] http://research.microsoft.com/ jiangli/portrait/

[9] Microsoft, http://msdn.microsoft.com/library/default. asp ?url=/li brary/en-us/dnanchor/html/ancrtc.asp

[10] Jonathan Rosenberg, A Data Format for Presence Using XML, IETF draft expired in 2000. http://www.iptel.org/info/ players/ietf/presence/outdated/draft-rosenberg-impp-pidf00. txt

[11] H. Sugano, et al., Presence Information Data Format (PIDF), RFC 3863, IETF, Aug. 2004.

[12] H. Schulzrinne, et al., RTP: A Transport Protocol for Real-Time Applications, RFC 1889, IETF, January. 1996. [13] J. Rosenberg, et al., STUN - Simple Traversal of User Datagram Protocol (UDP) Through Network Address Translators (NATs), RFC 3489, IETF, March 2003. [14] Jürgen Morhöfer's, GSM 06.10 lossy speech compression, http://kbs.cs.tu-berlin.de/ jutta/toast.html [15] Microsoft, Toll Quality Voice - Microsoft GSM, IETF Draft expired in 1999, http://www.opengroup.org/messaging/ vpim/specs/drafts/draft-ema-vpim-msgsm-00.txt

[16] PlanetaMessenger.org, http://planeta.sourceforge.net/

[17] YMSG Java API, http://jymsg9.sourceforge.net/

[18] Bart Lamot, JSkype API, http://www.lamot.net/jskype/, [19] Sun Microsystem, JSR 32: JAIN SIP API Specification, 28th Feb. 2005.

[20] U.S. National Institute of Standards and Technology (NIST), NIST-SIP 1.2, https://jain-sip.dev.java.net/

[21] Place Lab, www.placelab.org

[22] http://www.jsresources.org/

[23] http://www.jabber.org/

[24] Open Mobile Alliance, OMA Instant Messaging and Presence Service V1.2.1

[25] K. El-Khatib, et al., Support for Personal and Service Mobility in Ubiquitous Computing Environments, EuroPar 2003.

[26] Stefan Berger, et al, Ubiquitous computing using SIP, NOSSDAV'2003.

[27] Jason I. Hong, et al., An Architecture for PrivacySensitive Ubiquitous Computing, MobiSys2004.

[28] http://www.ineen.com/

[29] CounterPath Solutions, Inc., http://www.xten.com/

[30] http://en.wikipedia.org/wiki/OpenWrt 